\title{
Role of bronchoalveolar lavage in immunocompromised patients with pneumonia treated with a broad spectrum antibiotic and antifungal regimen
}

\author{
I A Hohenadel, M Kiworr, R Genitsariotis, D Zeidler, J Lorenz
}

\begin{abstract}
Background-In a retrospective study the value of bronchoalveolar lavage (BAL) in the diagnosis of pneumonia was investigated in 95 immunocompromised patients suffering from haematological disorders and receiving a regimen of broad spectrum antibiotics and antifungal agents (BSAR). Methods-With the exception of four afebrile patients, all had fever, raised $C$ reactive protein (CRP) levels, and new infiltrates visible on chest radiography. All patients underwent BAL to identify the organism causing the pneumonia and surveillance cultures were performed regularly for pathogens at different sites. Following classification of the isolates, patients with positive cultures were subdivided into two groups, pathogenic or contaminated. We investigated whether relevant pathogens were cultured only from the BAL fluid and whether they were susceptible to BSAR.
\end{abstract}

Department of Pulmonary Medicine, Academic Teaching

Hospital

Köln-Merheim,

D-51109 Köln,

Germany

I A Hohenadel

D Zeidler

Department of Pulmonary Medicine, University Hospital, Mainz, Germany

M Kiworr

Department of Microbiology, University Hospital, Mainz, Germany

R Genitsariotis

Department of

Pulmonary Medicine, Academic Teaching

Hospital, Lüdenscheid,

Germany

J Lorenz

Correspondence to: Dr I A Hohenadel iris@hohenadel.com

Received 24 February 2000 Returned to authors 17 April 2000

Revised version received 6 October 2000

Accepted for publication

27 October 2000
Results-Although 77 of the 95 patients were thrombocytopenic, bleeding during BAL occurred in only $15 \%$ of all patients. Ten days after the procedure the fever improved in $88 \%$ of patients, radiographic findings improved in $71 \%$, and CRP levels improved in $75 \%$ of patients; $22 \%$ of patients died within 28 days. Pathologically relevant isolates were found in $65 \%$ of all patients. Respiratory pathogens were detected only in the BAL fluid of 29 of the 95 patients $(35 \%$ Gram positive species, $40 \%$ Gram negative species, $11 \%$ Mycobacterium, $11 \%$ fungi, and $3 \%$ cytomegalovirus). In 16 of these 29 patients (55\%) the pathogens cultured only from the BAL fluid were resistant to treatment. Pathogens detected only in the BAL fluid were not susceptible to a standard broad spectrum antibiotic and antifungal regimen including teicoplanin, ceftriaxon, tobramycin, and amphotericin B in 12 of the 29 patients $(41 \%)$.

Conclusions-Our data suggest that 12 patients were treated with broad spectrum antimicrobial agents which were not directed at the appropriate organism on in vitro sensitivity tests without BAL. BAL is a relatively safe procedure in the diagnosis of pneumonia, supplying important information in immunocompromised patients as well as in immunocompromised patients receiving BSAR.

(Thorax 2001;56:115-120)
Keywords: bronchoalveolar lavage; pneumonia; immunosuppression

Pneumonia is a major cause of death in immunocompromised patients with haematological disorders; the mortality associated with untreated Legionella pneumophila infection in immunocompromised patients has been reported to be $80 \% .{ }^{1}$ Identification of the causative pathogen and initiation of effective treatment is therefore essential. We have investigated the diagnostic value of bronchoalveolar lavage (BAL) in 95 immunocompromised patients with haematological disorders and clinical signs of acute pneumonia, comparing BAL with non-invasive procedures such as surveillance cultures at different sites. We determined whether the BAL findings are associated with the therapeutic outcome for immunocompromised patients routinely receiving a broad spectrum antibiotic and antifungal regimen (BSAR). All BAL related complications were documented, including those observed for patients with thrombocytopenia. This study assesses the effect of the severity of immunodeficiency on a spectrum of pathogens as well as on the clinical course of pneumonia.

\section{Methods}

The retrospective study took place in the Department of Pulmonary Medicine in the University Hospital in Mainz, Germany over a period of 4 years and 2 months. All patients with haematological disorders and neutropenia were enrolled in the study if they presented with signs of acute pneumonia. The median (SD) age of the 95 immunocompromised patients (68 men, 27 women) was 49.4 (14.5) years. Underlying diseases included acute myeloid leukaemia (45), acute lymphatic leukaemia (10), chronic myeloid leukaemia (10), non-Hodgkin's lymphoma (10), myelodysplastic syndrome (7), Hodgkin's disease (5), chronic lymphatic leukaemia (3), aplastic anaemia or agranulocytosis (5). Patients were divided into two groups according to the neutrophil granulocyte count: group A included 71 patients with a neutrophil granulocyte count of less than $1000 / \mu 1$ and group B included 24 patients with a neutrophil granulocyte count of 1000-3500/ $\mathrm{l}$. With the exception of four afebrile patients, all patients presented with respiratory symptoms (such as dyspnoea and cough), fever, raised $C$ reactive protein (CRP) levels, and new infiltrates as shown by chest 
radiography. BSAR was administered to all those except the patients with renal dysfunction for whom the antibiotic and antifungal prescription was changed.

All patients underwent BAL in our department. After inspection of the transbronchial tree to the subsegmental level, the bronchoscope was wedged into a subsegment bronchus in the area with the most marked radiological abnormality or, in the presence of diffuse infiltration, into the lingula or middle lobe. ${ }^{2} \mathrm{BAL}$ was performed according to a standard technique $^{3}$ using 5-7 $20 \mathrm{ml}$ aliquots of normal saline. Suctioning after each bolus yielded a total of $40-100 \mathrm{ml}$ of fluid. Aliquots of lavage fluid were submitted for microscopic examination (Gram, acridin and acid-fast stains) and cultured for aerobic and anaerobic bacteria, fungi, mycobacteria, and viruses quantitatively. Standard culture methods were used. Direct immunofluorescence was employed for the detection of Legionella pneumophila as well as cultures and polymerase chain reaction in the last year of the study. For mycobacteria Ziehl Nielsen acid-fast stains and cultures were performed. Detection of cytomegalovirus was performed by immunofluorescence techniques and human cell cultures. For all pathogenic stains isolated antibiograms were primarily determined. Transbronchial biopsy specimens could only be taken from 11 patients; all the remaining patients had thrombocytopenia and/or coagulopathy. The biopsy specimens were taken from the area of interest or from the lower lobes in the presence of a diffuse infiltration. Only two patients underwent open lung biopsies using an invasive procedure. Radiographs were routinely taken during the 24 hours after the transbronchial biopsy specimens to rule out pneumothorax. Fever, laboratory parameters, radiographs, medication including preceding chemotherapy, and duration of neutropenia were recorded from admission to hospital to discharge. Cultures from BAL fluid, sputum, blood, urine, pharyngeal smears, and smears from other sites were performed regularly for all patients. Patients with positive cultures were subdivided following classification of the isolates into pathogenic or contaminated groups during the 14 days before and after BAL. While colonies of Legionella were considered to be pathogenic, coagulase negative Staphylococcus or Candida were considered to be pathogenic only when cultured several times from different sources in the presence of a consistent clinical course.

To determine the diagnostic value of BAL we investigated whether the respiratory pathogens were sensitive to the treatment as well as to a BSAR consisting of teicoplanin, ceftriaxon, tobramycin, and amphotericin B. This standard BSAR regimen was selected on the basis of our clinical experience and according to published recommendations. ${ }^{4}$

STATISTICAL ANALYSIS

The Wilcoxon test for two samples, the $\chi^{2}$ test, and Fisher's exact test (two sided) were used. Values are expressed as mean (SD). A value of $\mathrm{p}<0.05$ was considered significant. ${ }^{5}$

\section{Results}

PATIENT CHARACTERISTICS

Seventy one immunocompromised patients were allocated to group A (neutrophil granulocyte count less than $1000 / \mu \mathrm{l}$ ) and 24 to group B

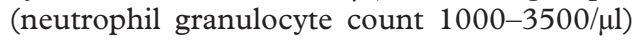
according to the severity of the underlying condition. The mean value of the lowest granulocyte count was 590/ $\mu$ l (range 0-3400/ $\mu 1)$. Seventy six patients had undergone chemotherapy during the six months preceding BAL (mean 23 days). Nine patients had received a bone marrow transplant. Admission to hospital was on average 18.7 days before BAL (range 0-70 days); 71 patients were admitted for a minimum of five days before the procedure. A total of 71 patients were therefore at risk of "hospital acquired pneumonia" with more virulent pathogens. Onset of fever was noted on average 9.4 days and pulmonary infiltrates were detected 7.7 days preceding BAL. There were no significant differences between groups $\mathrm{A}$ and $\mathrm{B}$ with respect to age, sex, mean duration of BAL, preceding chemotherapy, or the ratio of patients with chemotherapy and clinical parameters.

Intravenous antibiotics and/or antifungal agents were administered to 82 of the 95 patients (86\%) 9.6 days before BAL. In 80 of the 95 patients $(84 \%)$ the antibiotic and/or antifungal regimen was changed during the 10 days after the procedure as a result of BAL findings and/or clinical observations. In 73 of the 95 patients antifungal agents were given intravenously. Most patients received the described standard BSAR regimen. There was no significant difference in the percentage of patients treated with BSAR and the duration of antibiotic therapy between groups A and B. Group A included more patients with underlying acute myeloid leukaemia $(p=0.004)$, while the number of patients with non-Hodgkin's lymphoma $(p=0.015)$ and Hodgkin's disease $(p=0.014)$ was greater in group B. Surprisingly, there was no difference in the time from the onset of neutropenia to BAL between the two groups.

COMPLICATIONS OF BAL

No episodes of collapse, allergic reaction to anaesthesia, or pneumothorax occurred during or after bronchoscopy. Seventy seven of the 95 patients $(81 \%)$ were thrombocytopenic (median platelet count $38000 / \mu \mathrm{l})$. The ratio of patients with thrombocytopenia was significantly higher in group A $(\mathrm{p}=0.002)$. The platelet count was below $50000 / \mu 1$ in 58 patients and below $20000 / \mu 1$ in 16 . No patient had major bleeding complications. Minor limited bleeding was observed in $15 \%$ of the patients. Twenty $\mathrm{ml}$ norepinephrine (noradrenaline) were administered locally to only one patient with a normal platelet count to stop nonhaemodynamic bleeding after transbronchial biopsies. One of the 11 patients who underwent a transbronchial biopsy developed minimal bleeding after the procedure. Additional anaesthetics or sedatives were administered to seven patients with severe coughing. Bronchoscopic examination could not be carried out in 
Table 1 Characteristics of patients who died during the 28 days following bronchoalveolar lavage (BAL)

\begin{tabular}{|c|c|c|c|c|c|}
\hline Patient no & Group & Isolates from BAL fluid & Isolates from other sources & $\begin{array}{l}\text { Death (days } \\
\text { after } B A L)\end{array}$ & Additional comments \\
\hline 1 & B & Enterococcus faecalis & Enterococcus (ph) & 4 & \\
\hline 5 & A & $\begin{array}{l}\text { Coagulase negative } \\
\text { Staphylococcus }\end{array}$ & $\begin{array}{l}\text { Coagulase negative Staphylococcus } \\
(\mathrm{ph}, \mathrm{b})\end{array}$ & 15 & \\
\hline 10 & A & $\begin{array}{l}\text { Multiresistant Staphylococcus; } \\
\text { Enterococcus faecalis }\end{array}$ & $\begin{array}{l}\text { Multiresistant Staphylococcus (b); } \\
\text { Staphylococcus aureus (b); } \\
\text { Aspergillus (sp, u); Candida (sp, } \\
\text { rare) }\end{array}$ & 5 & \\
\hline 12 & A & Legionella pneumophila & Negative & 16 & \\
\hline 14 & B & Acinetobacter; $E$ hermani & Candida (sp, ph) & 18 & \\
\hline 24 & B & Candida & Candida (ph, sp) & 21 & $\begin{array}{l}\text { Patient refused } \\
\text { antifugal therapy after } 3 \\
\text { days of treatment }\end{array}$ \\
\hline 27 & A & Negative & Negative & 6 & \\
\hline 28 & A & Legionella pneumophila & $\begin{array}{l}\text { Acinetobacter }(\mathrm{sp}) ; \text { Staphylococcus } \\
\text { aureus }(\mathrm{sp}, \mathrm{ph})\end{array}$ & 24 & \\
\hline 33 & B & M tuberculosis & Negative & 3 & \\
\hline 34 & A & Negative & Aspergillus (sp); Candida (sp, $\mathrm{ph}, \mathrm{f})$ & 3 & \\
\hline 39 & A & $\begin{array}{l}\text { Stenotrophomonas maltophilia; } \\
\text { Capnocytophaga; Staphylococcus }\end{array}$ & Staphylococcus $(\mathrm{ph})$ & 5 & \\
\hline 44 & B & Aspergillus fumigatus; Candida & $\begin{array}{l}\text { Aspergillus fumigatus (sp); Candida } \\
\text { (sp) }\end{array}$ & 20 & \\
\hline 47 & A & $\begin{array}{l}\text { Aspergillus fumigatus; Candida; } \\
\text { Streptococcus; Lactobacillus }\end{array}$ & Candida $(\mathrm{sp}, \mathrm{f})$ & 15 & $\begin{array}{l}\text { Necropsy: fungal } \\
\text { pneumonia }\end{array}$ \\
\hline 48 & A & Multiresistant Staphylococcus & Negative & 26 & \\
\hline 60 & B & Negative & Negative & 2 & \\
\hline 64 & A & Enterococcus faecalis & Negative & 12 & \\
\hline 70 & B & Candida & $\begin{array}{l}\text { Candida }(\mathrm{ph}) ; \text { Klebsiella } \\
\text { pneumoniae }(\mathrm{ph})\end{array}$ & 1 & \\
\hline 71 & A & Negative & Negative & 20 & $\begin{array}{l}\text { ARDS, pneumothorax } \\
\text { related to mechanical } \\
\text { ventilation }\end{array}$ \\
\hline 78 & B & Candida & Candida $(\mathrm{ph}, \mathrm{f})$ Staph aureus $(\mathrm{ph})$ & 19 & \\
\hline 90 & B & $\begin{array}{l}\text { Cytomegalovirus; Candida; } \\
\text { Staphylococcus; Lactobacillus }\end{array}$ & $\begin{array}{l}\text { Candida }(\mathrm{u}, \mathrm{f}, \mathrm{sp}, \mathrm{ph}) \\
\text { Staphylococcus }(\mathrm{sp}, \mathrm{ph})\end{array}$ & 7 & $\begin{array}{l}\text { Necropsy: } \\
\text { cytomegalovirus } \\
\text { pneumonia }\end{array}$ \\
\hline
\end{tabular}

$\mathrm{ph}=$ pharyngeal smears $; \mathrm{sp}=$ sputum $; \mathrm{b}=$ blood $\mathrm{f}=$ faeces $\mathrm{u}=$ urine.

one patient with persistent coughing. There were no differences in coughing or bleeding episodes between the two groups and no correlation was established between the platelet count and bleeding.

CLINICAL COURSE

Ten days after BAL $88 \%$ of the patients were afebrile and the radiographic findings and CRP levels were improved or unremarkable in $71 \%$ and $75 \%$, respectively. A positive outcome (decline of fever together with improved radiography) occurred in $55 \%$ of patients, a negative outcome (either no decline or increase in temperature or unchanged radiography) occurred in $23 \%$ of patients, and $22 \%$ of patients died during the 28 days following BAL (table 1). There were no significant differences in these parameters between groups $\mathrm{A}$ and $\mathrm{B}$, although the number of patients who died during the 28 days after BAL was significantly higher in group $B(p=0.02)$. No significant correlation was found between the outcome and either the type of pathogen or the age of the patient. Two of the three patients in whom Legionella pneumophila was cultured only in the BAL fluid died within 28 days of the procedure.

CYTOLOGICAL EXAMINATION OF BAL FLUID AND TRANSBRONCHIAL BIOPSY SPECIMENS

An additional cytological examination of the BAL fluid was performed at the local department of pathology for 66 of the 95 patients. The results revealed signs of inflammation in 30 of the 66 patients and malignancy was seen in three; hyphae consistent with necroscopic findings confirmed Candida pneumonia in two patients and multiple granulomas with Langerhans' cells suspected of Mycobacterium tuberculosis infection were seen in one. In 11 of the 95 patients transbronchial biopsy specimens were taken and malignant lymphoma cells were found in three patients (underlying disease non-Hodgkin's lymphoma in all three), peribronchial fibrosis was noted in one patient, and signs of a graft-versus-host reaction were seen in another patient. One transbronchial biopsy specimen confirmed the BAL finding of $\mathrm{Myco}-$ bacterium tuberculosis. In three patients in whom no pathogens were found in the BAL fluid, blood, sputum, or other sites, signs of malignancy were observed in the transbronchial biopsy specimens of two and on cytological examination of the BAL fluid in one; malignancy was therefore the most likely cause of the pulmonary infiltrates and fever in these three patients. Bronchoscopy therefore provided important information in the diagnosis of pulmonary infiltrates in 65 of the 95 patients $(68.4 \%)$.

COMPARISON OF BAL FINDINGS WITH NECROPSIES AND OPEN LUNG BIOPSY SPECIMENS

Necroscopic examination confirmed the BAL findings in two patients with a diagnosis of Candida pneumonia, in one patient with squamous cell carcinoma and concomitant bronchopneumonia, and in one patient with severe interstitial cytomegalovirus pneumonia. Open lung biopsy demonstrated aspergillosis with a cavitation filled with hyphae of Aspergillus fumigatus (aspergilloma) and accompanying bronchopneumonia in two patients in whom BAL had detected no evidence of Aspergillus. In 
view of the fact that open lung biopsy is an invasive procedure, it is rarely performed in patients with leukopenia because of concomitant thrombocytopenia and the risk of bleeding. A statistical analysis could therefore not be performed. A strong correlation was established between the BAL fluid and necroscopic findings, although pathogens such as Aspergillus are not detected by BAL if they are localised in cavitations.

\section{MICROBIOLOGICAL FINDINGS}

Surveillance cultures at different sites were regularly performed for 95 immunocompromised patients with signs of acute pneumonia. Positive cultures were found in BAL fluid $(91 \%)$, blood $(13 \%)$, sputum $(93 \%)$, urine $(12 \%)$, pharyngeal smears $(88 \%)$, and other sources including smears obtained from central vein catheters, endotracheal tubes, and pleura fluid $(47 \%)$. Pathogenically relevant isolates from the respective sources were detected in BAL fluid $(65 \%)$, blood (11\%), sputum $(70 \%)$, urine $(6 \%)$, pharyngeal smears $(59 \%)$, and smears from other sources $(30 \%)$. The results of cultured pathogenic colonies from BAL fluid are presented in table 2.

In 62 of the 95 patients $(65 \%)$ a pathogen likely to be responsible for pneumonia was cultured from the BAL fluid. In 36 of these 62 patients $(58 \%)$ microbiological findings showed the pathogens from the BAL fluid to be resistant to the treatment given. The ratio of patients receiving inadequate treatment was significantly higher $(p=0.006)$ in group $B$ than in group A. If all 62 patients had undergone treatment with a BSAR consisting of teicoplanin, ceftriaxon, tobramycin, and amphotericin $\mathrm{B}$, the treatment would have been ineffective in 20 of the 62 patients (32\%) in whom pathogens were detected in the BAL fluid. The following pathogens were resistant to BSAR: Mycobacterium tuberculosis or $M$ xenopii, Capnocytophaga, multiresistant Staphylococcus, Staphylococcus aureus, Stenotrophomonas, Acinetobacter, Legionella, Chlamydia, and cytomegalovirus.

In 29 of the 95 patients $(31 \%)$ the organism responsible for pneumonia was detected only in the BAL fluid (35\% Gram positive species, 40\% Gram negative species, 11\% Mycobacterium, $11 \%$ fungi, $3 \%$ cytomegalovirus). Micro-

Table 2 Pathogens cultured from bronchoalveolar lavage (BAL) fluid in 95 immunocompromised patients with pneumonia

\begin{tabular}{lcll}
\hline Pathogens & $\begin{array}{l}\text { Frequency } \\
(\%)\end{array}$ & $\begin{array}{l}\text { Classification } \\
\text { of pathogens }\end{array}$ & $\begin{array}{l}\text { Frequency } \\
(\%)\end{array}$ \\
\hline Staphylococcus & $23(25.3 \%)$ & Gram positive & $40.7 \%$ \\
Enterococcus & $9(9.9 \%)$ & & \\
Streptococcus & $3(3.3 \%)$ & & \\
Saureus & $2(2.2 \%)$ & & \\
Acinetobacter & $1(1.1 \%)$ & Gram negative & $16.5 \%$ \\
Capnocytophaga & $4(4.4 \%)$ & & \\
Chlamydia & $2(2.2 \%)$ & & \\
Enterobacteriacea & $2(2.2 \%)$ & & \\
Haemophilus & $2(2.2 \%)$ & & $6.6 \%$ \\
Legionella & $3(3.3 \%)$ & & $35.2 \%$ \\
Pseudomonas & $1(1.1 \%)$ & & $1.1 \%$ \\
Mycobacterium & $6(6.6 \%)$ & Mycobacteria \\
Candida & $25(27.5 \%)$ & Fungi & \\
Aspergillus & $7(7.7 \%)$ & & \\
Cytomegalovirus & $1(1.1 \%)$ & Viruses & \\
\hline
\end{tabular}

biological findings showed that pathogens cultured only in the BAL fluid were resistant to the treatment given in 16 of the 29 patients (55\%). Thus, without BAL, $44 \%$ of the patients in group A and all of the patients in group B would have been treated with antimicrobial agents which were not directed at the appropriate organism $(\mathrm{p}=0.02)$. If all 29 patients had received a BSAR consisting of teicoplanin, ceftriaxon, tobramycin, and amphotericin $\mathrm{B}$, the regimen would have been ineffective in 12 of the 29 patients (41\%). With BSAR, $13 \%$ of all patients would have received insufficient treatment.

There were no differences between the two groups with regard to positive cultures or pathogens with the exception of Candida detected in BAL fluid. The significantly lower incidence of Candida in group A than in group B $(p=0.005)$ may be because antifungal therapy was administered to more patients in this group. Antifungal agents were given to $61 \%$ of patients in group A and to only $25 \%$ of patients in group B. No significant correlation was observed between the individual pathogen and the outcome or the severity of immunosuppression or duration of neutropenia with the exception of mycobacteria which were associated with a longer duration of neutropenia $(p=0.03)$. We could not establish a relationship between the location of pathogens and the susceptibility to antibiotics or antifungal agents and the outcome of treatment.

\section{Discussion}

We have compared the diagnostic value of BAL as an invasive procedure in 95 immunocompromised patients with haematological disorders and signs of pneumonia with non-invasive procedures such as surveillance cultures at different sites. BAL and sputum had the highest diagnostic yields. However, in assessing this result consideration has to be given to the fact that, in our study, only one culture was taken from the BAL fluid compared with multiple sputum samples (up to 12) and samples from different sources. It is also of interest to determine whether pathogens observed in BAL fluid were also present in sputum. Our findings show that organisms responsible for pneumonia were detected only in the BAL fluid in 29 of the 95 patients $(31 \%)$ while all other samples, including sputum, had no diagnostic yield. The causative organisms were therefore not detected by less invasive procedures in 29 patients. Sputum was found to be useful in diagnosis in only $14 \%$ of the immunocompromised patients with pneumonia compared with $30 \%$ bronchial washings, $38 \%$ brushings, and $73 \%$ transbronchial biopsy specimens reported by Chopra. ${ }^{6}$ Transbronchial biopsy specimens were of diagnostic value in seven of 11 patients $(64 \%)$ and relevant pathogens were cultured from BAL fluid in $65 \%$ of all patients in our study.

The value of BAL in the diagnosis of pneumonia in immunocompromised patients with different underlying diseases cited in the literature ranges from $33 \%^{7}$ to $83 \% .^{8}$ A diagnostic yield of $81 \%{ }^{9}$ has been reported in 
patients with and without immunosuppression, with a yield ranging from $60 \%{ }^{10}$ to $66 \%{ }^{11}$ in patients with haematological disorders exclusively and of only $50 \%{ }^{12}$ in patients with a bone marrow transplant. Young reported a positive result for BAL in the diagnosis of pneumonia or malignancy in $93 \%$ of all patients. ${ }^{13}$

Most of the studies on pneumonia in immunocompromised patients do not differentiate between the different underlying diseases. While Streptococcus pneumoniae is the most common pathogen responsible for pneumonia in immunocompetent patients ${ }^{14}$ and Pneumocystis carinii is one of the main causes of pneumonia in HIV infected patients, ${ }^{15}{ }^{16}$ von Eiff ${ }^{11}$ reported Gram negative bacteria (in 38 of 90 patients) and fungi (in 34 of 90 patients) to be the most common pathogens in patients with haematological malignancies and pneumonia. We found both Gram positive (35\%) and Gram negative species $(40 \%)$ as well as fungi and mycobacteria (11\% each) in the BAL fluid.

The most difficult problem encountered in this study was deciding whether a positive culture was consistent with a pathogen responsible for pneumonia or whether it was caused by contamination of the nasopharynx. In particular, for Candida and coagulase negative Staphylococcus there are no firm recommendations as to whether a positive culture in the BAL fluid of immunocompromised patients with signs of pneumonia necessitates treatment. A distinct increase in infections in immunocompromised patients caused by multiresistant or methicillin resistant coagulase negative Staphylococcus has been described in the literature. ${ }^{17}$ Immunocompromised patients are known to develop severe pneumonia. ${ }^{18}$ The diagnosis of coagulase negative Staphylococcus infection (not contamination) is suggested when the organism is cultured from normally "sterile" materials such as BAL fluid or blood, ${ }^{12}$ when it is cultured several times in different blood samples, ${ }^{19}$ or when it is resistant to five or more different antibiotics. ${ }^{20}$ In this study we accepted coagulase negative Staphylococcus as a pathogen only when it was detected in at least two different sources or twice in different samples taken on different days and when it was resistant to at least five antibiotics.

Candida is a common organism of the mucous membrane, ${ }^{1}$ but damage to the mucous membrane resulting from immunosuppression leads to disseminated severe infection. ${ }^{21}$ We therefore accepted Candida as a pathogen in immunocompromised patients with pneumonia and a concordant clinical course only when it was detected in different sources, or twice in different samples taken on different days, or in combination with a positive cytological result. ${ }^{22}$

There are no definite recommendations for the diagnosis of an Aspergillus infection and the diagnosis of invasive pulmonary aspergillosis can only be established by biopsy. ${ }^{23}$ However, this cannot be performed in most immunocompromised patients because of coexisting thrombocytopenia. Although contamination of BAL fluid cannot be excluded, it is nevertheless a valuable diagnostic tool for
Aspergillus pneumonia. ${ }^{24}$ In an investigation performed by Stover et al ${ }^{8}$ BAL had a diagnostic yield in $83 \%$ of all patients with invasive pulmonary aspergillosis confirmed by open lung biopsy, transbronchial biopsies, and necropsy. Von Eiff et $a l^{25}$ reported a sensitivity of $87 \%$ and a specificity of $100 \%$ for BAL. A single positive sputum culture for Aspergillus has to be viewed critically, while a positive BAL culture in immunocompromised patients should not be underestimated as contamination but should, in view of a mortality rate of approximately $14.8 \%$ reported for invasive pulmonary aspergillosis, ${ }^{26}$ serve as an indication for the early onset of treatment with amphotericin B. ${ }^{26}$ Aspergillus was cultured from BAL fluid in five of seven patients in our study. The two patients in whom BAL was not diagnostic were subsequently shown to have an aspergilloma which cannot be detected by BAL. One patient with a positive sputum culture for Aspergillus and a negative BAL finding underwent open lung biopsy with no evidence of invasive pulmonary aspergillosis, confirming the accuracy of the BAL findings obtained by our investigation. Marra et $a l^{10}$ found that Aspergillus was responsible for pneumonia in $20 \%$ of all patients with lymphoma or leukaemia. Analysis of the 62 patients in our study in whom a pathogen was cultured from the BAL fluid showed that Aspergillus was responsible for pneumonia in $11 \%$ of patients.

Mycobacteria other than tuberculosis (MOTT) - for example, $M$ xenopii-are considered to be pathogenic in immunocompromised patients when cultured in a sterile medium such as BAL fluid or found in a biopsy specimen compatible with the clinical course. MOTT detected in a "non-sterile" medium such as sputum were considered to be pathogenic when more than 100 colonies could be seen in each of four different samples. ${ }^{27}$ Although $M$ xenopii may occur in immunocompetent subjects as a contaminant, this is not the case in immunocompromised patients. ${ }^{27}$

An important question remains if BAL findings are to be of therapeutic use in patients with BSAR, most of whom routinely receive this treatment. We detected pathogens responsible for pneumonia in the BAL fluid of 62 of the 95 patients $(65 \%)$. Microbiological findings showed that the pathogens cultured from the BAL fluid were resistant to treatment in $58 \%$ of patients and to standard BSAR consisting of teicoplanin, ceftriaxon, tobramycin, and amphotericin B in $32 \%$ of all patients. There are few data in the literature to indicate whether BSAR consisting of teicoplanin, ceftriaxon, tobramycin, and amphotericin B is effective in the treatment of pathogens, or if the treatment regimen is changed after BAL. Marra et $a l^{10}$ reported that the standard BSAR was changed in $18 \%$ of patients as a result of BAL findings. Pathogens from the BAL fluid of patients with hospital acquired pneumonia were resistant to BSAR in $67 \%$ of cases $^{10}$ and pathogens cultured from the BAL fluid of patients with immunosuppression were resistant in $43 \%$ of 
cases. ${ }^{9}$ Empirical antimicrobial treatment was verified by bronchoscopy in 25 of 90 patients and was successfully changed in 34 patients. ${ }^{11}$ Our findings showed that causative pathogens detected exclusively in the BAL fluid in 29 of our 95 patients were resistant to the treatment given in 16 patients who would therefore have received inadequate treatment without BAL. Treatment with standard BSAR including teicoplanin, ceftriaxon, tobramycin, and amphotericin B would have been ineffective in 12 of 29 patients $(41 \%)$ with pathogens detected in BAL fluid only.

Complications related to BAL consisted of minor self-limiting bleeding in $14.7 \%$ of patients; only one patient had more severe, non-haemodynamically relevant bleeding, although 77 of 95 patients were thrombocytopenic. No pneumothorax occurred. Studies on BAL related complications with a particular focus on immunocompromised patients have reported self-limiting bleeding, bronchial spasm, and coughing in $33 \%$ of patients. ${ }^{28}$ Von Eiff $e t a l^{25}$ noted that complications such as pneumothorax, respiratory symptoms requiring treatment in the intensive care unit, and self-limiting bleeding occurred in $5 \%$ of patients with haematological disorders, transplant recipients, or those with AIDS. Zavala ${ }^{29}$ reported bleeding following bronchoscopy in $26 \%$ of immunocompromised patients.

Thus, BAL may be considered to be a relatively safe procedure even for patients with a low platelet count when compared with the risk of untreated or unsuccessfully treated pneumonia in patients with neutropenia. In view of the cost of bronchoscopy (about 360 German marks or 180 Euro), ${ }^{30}$ this is a very small sum of money compared with the costs of a longer stay in hospital for severely ill patients. However, the question of cost effectiveness goes beyond the scope of this study. Our results suggest that BAL may be essential in the diagnosis of pneumonia in immunocompromised patients.

In conclusion, we have shown that the causative organisms were not detected by less invasive tools in 29 of 95 patients $(31 \%)$ and that the pathogens found were resistant to BSAR in 12 of the 29 patients $(41 \%)$. Even though 77 of 95 patients were thrombocytopenic, no major bleeding occurred. BAL is therefore a relatively safe procedure in the diagnosis of pneumonia in immunocompromised patients and in those treated with BSAR.

1 Edelstein H. Antimicrobial chemotherapy for Legionnaire's disease: a review. Clin Infect Dis 1995;2-3:265-76.

2 Reynolds HY. State of art: bronchoalveolar lavage. Am Rev Respir Dis 1987;135:250-63.

3 Hunninghake GW, Gadek JE, Kawanami O, et al. Inflammatory and immune processes in the human lung in health and disease: evaluation by bronchoalveolar lavage. $A m \mathcal{F}$ Clin Pathol 1979;97:149-206. 4 Maschmeyer G, Link H, Hiddemann W, et al. Empirische Med Klin 1994;89:114-23.

5 The GLM procedure. SAS user's guide. Cary, NC: SAS Institute, 1985.

6 Chopra SK, Mohsenifar Z. Fiberoptic bronchoscopy in diagnosis of opportunistic lung infections. West $\mathcal{f} \mathrm{Med}$ 1979;131:4-8.

7 Martin WJ, Smith TF, Brutinel WM, et al. Role of bronchoalveolar lavage in the assessment of opportunistic pulmonary infections: utility and complications. Mayo Clin Proc 1987;62:549-57.

8 Stover DE, Muhammad BZ, Steven IH, et al. Bronchoalveolar lavage in diagnosis of diffuse pulmonary infiltrates in the immunosuppressed host. Ann Intern Med 1984;101: in the

9 Dalhoff K, Braun J, Hollandt H, et al. Diagnostic value of bronchoalveolar lavage in patients with opportunistic and nonopprtunistic bacterial pneumonia. Infection 1993;21: 291/17-296/22.

10 Marra R, Pagano L, Pagliari G, et al. The yield of bronchoalveolar lavage in the etiologically diagnosis of pneumonia in leukemia and lymphoma patients. Eur f Haematol 1993; 51:256-8.

11 Von Eiff $\mathrm{M}$, Zuhlsdorf $\mathrm{M}$, Roos $\mathrm{N}$, et al. Pulmonary infiltrates in patients with hematologic malignancies: clinical usefulness of non-invasive bronchoscopic procedures. cal usefulness of non-invasive bron

12 Cordonnier C, Bernaudin JF, Fleury J, et al. Diagnostic yield of bronchoalveolar lavage in pneumonitis occurring after allogenic bone marrow transplantation. Am Rev Respir Dis 1985;132:1118-23.

13 Young JA, Hopkin JM, Cuthbertson WP. Pulmonary infiltrates in immunocompromised patients. Diagnosis by cytologic examination of bronchoalveolar lavage fluid. $\mathcal{7}$ Clin Pathol 1984;37:390-7.

14 Østergaard L, Andersen PL. Etiology of community acquired pneumonia. Chest 1993;104:1400-7.

15 Lerner CW, Tapper ML. Opportunistic infection complicating acquired immune deficiency syndrome. Medicine 1984; 63:155-64.

16 Müller IA, Gloger J, Genitsariotis R, et al. The role of bronchoalveolar lavage in HIV-infected patients with pneumonia in comparison to non-invasive procedures. Am $\mathcal{F}$ monia in comparison to non-invasive
Respir Crit Care Med 1997;155:A-472

17 Vincent JL, Bihari DJ, Suter PM, et al. The prevalence of nosocomial infections in intensive care units in Europe. fAMA 1995;274:639-44.

18 Jones RN. Impact of changing pathogens and antimicrobial susceptibility patterns in the treatment of serious infections in hospitalized patients. Am F Med 1996;100:3-12S

19 Rubio M, Palau L, Vivas JR, et al. Predominance of gram-positive microorganisms as a cause of septicemia in patients with hematological malignancies. Infect Control Hosp Epidemiol 1994;15:101-4.

20 Christensen GD, Bisno AL, Parisi JT, et al. Nosocomial septicemia due to multiply antibiotic-resistant Staphylococcus epidermidis. Ann Intern Med 1982;96:1-10.

21 Rosenow EC, Wilson WR, Cockerill FR. Pulmonary disease in the immunocompromised host. Mayo Clin Proc 1985;60: 473-87.

22 Saito H, Anaissie EJ, Morice RC, et al. Bronchoalveolar lavage in the diagnosis of pulmonary infiltrates in patients with acute leukemia. Chest 1988;94:745-9.

23 Maschmeyer G, Link H, Hiddemann W, et al. Pulmonary nfiltrations in febrile patients with neutropenia. Cancer 1994;73:2296-304.

24 Kahn FW, Jones JM, England DM. The role of bronchoalveolar lavage in the diagnosis of invasive pulmonary aspergillosis. Am f Clin Pathol 1986;86:518-23.

25 von Eiff M, Steinemann R, Roos N, et al. Pneumonien bei abwehrgeschwächten Patienten: Stellenwert nicht bioptischer bronchoskopischer Untersuchungsverfahren in der Erregerdiagnostik. Klin Wochenschr 1990;68:372-9.

26 Yu VL, Muder RR, Poorsatter A. Significance of isolation of Aspergillus from the respiratory tract in diagnosis of invasive pulmonary aspergillosis. Results from a three-year prospective study. Am F Med 1986;81:249-54.

27 Tenholder MF, Moser RJ, Tellis CJ. Mycobacteria other than tuberculosis. Pulmonary involvement in patients with acquired immunodeficiency syndrome. Arch Intern Med 1988;148:953-6

$28 \mathrm{Kahn}$ WF, Jones JM. Analysis of bronchoalveolar lavage specimens from immunocompromised patients with a protocol applicable in the microbiology laboratory. 7 Clin Microbiol 1988;26:1150-5.

29 Zavala DC. Pulmonary hemorrhage in fiberoptic transbronchial biopsy. Chest 1976;70:584-8.

30 Broglie MG, Pranschke-Schade S, Schade H-J. In: Gebührenhandbuch. Kommentar für Ärzte. Wiesbaden: Medical Tribune Verlagsgesellschaft mbH, 1996. 